\title{
Transient four-wave mixing in T-shaped GaAs quantum wires
}

\author{
Langbein, Wolfgang Werner; Gislason, Hannes; Hvam, Jørn Märcher
}

Published in:

Physical Review B Condensed Matter

Link to article, DOI:

10.1103/PhysRevB.60.16667

Publication date:

1999

Document Version

Publisher's PDF, also known as Version of record

Link back to DTU Orbit

Citation $(A P A)$ :

Langbein, W. W., Gislason, H., \& Hvam, J. M. (1999). Transient four-wave mixing in T-shaped GaAs quantum wires. Physical Review B Condensed Matter, 60(24), 16667-16674. https://doi.org/10.1103/PhysRevB.60.16667

\section{General rights}

Copyright and moral rights for the publications made accessible in the public portal are retained by the authors and/or other copyright owners and it is a condition of accessing publications that users recognise and abide by the legal requirements associated with these rights.

- Users may download and print one copy of any publication from the public portal for the purpose of private study or research.

- You may not further distribute the material or use it for any profit-making activity or commercial gain

- You may freely distribute the URL identifying the publication in the public portal 


\title{
Transient four-wave mixing in T-shaped GaAs quantum wires
}

\author{
W. Langbein, ${ }^{*}$ H. Gislason, ${ }^{\dagger}$ and J. M. Hvam ${ }^{\ddagger}$ \\ Mikroelektronik Centret, The Technical University of Denmark, Building 345 east, DK-2800 Lyngby, Denmark
}

(Received 5 May 1999)

\begin{abstract}
The binding energy of excitons and biexcitons and the exciton dephasing in T-shaped GaAs quantum wires is investigated by transient four-wave mixing. The T-shaped structure is fabricated by cleaved-edge overgrowth, and its geometry is engineered to optimize the one-dimensional confinement. In this wire of 6.6 $\times 24 \mathrm{~nm}^{2}$ size, we find a one-dimensional confinement of more than $20 \mathrm{meV}$, an inhomogeneous broadening of $3.4 \mathrm{meV}$, an exciton binding energy of $12 \mathrm{meV}$, and a biexciton binding energy of $2.0 \mathrm{meV}$. A dispersion of the homogeneous linewidth within the inhomogeneous broadening due to phonon-assisted relaxation is observed. The exciton acoustic-phonon-scattering coefficient of $6.1 \pm 0.5 \mu \mathrm{eV} / \mathrm{K}$ is larger than in comparable quantum-well structures. [S0163-1829(99)04147-8]
\end{abstract}

\section{INTRODUCTION}

The realization of one-dimensional (1D) semiconductor nanostructures with large confinement energies and an enhanced spatial overlap of the electron and hole wave functions is of importance for their optoelectronic device applications. A variety of different fabrication techniques such as $e$-beam lithography, ${ }^{1}$ growth on misoriented substrates (Serpentine superlattices ${ }^{2}$ ), or prepatterned substrates (V-groove quantum wires ${ }^{3}$ ) have been demonstrated. Another possibility is the fabrication of a T-shaped intersection of two quantum wells (QWs), as was proposed by Chang, Chang, and Esaki. ${ }^{4}$ At the intersection, a bound quantum wire state $(\mathrm{T}$ QWR state) forms due to the reduction of the quantization energy. Such structures can be realized in the $\mathrm{Al}_{x} \mathrm{Ga}_{1-x} \mathrm{As}$ material system by the cleaved-edge overgrowth technique, ${ }^{5-9}$ where both confinement dimensions are controlled by the high accuracy of the molecular-beam epitaxy (MBE). The photoluminescence of these structures, attributed to the T-QWR state transition, was demonstrated to originate from the $\mathrm{T}$ intersections by optical near-field spectroscopy. ${ }^{10}$ Optically and electrically pumped lasing of the T-QWR transition was observed and attributed to excitonic processes. ${ }^{11,12}$ Large exciton binding energies ${ }^{13}$ and optical anisotropy ${ }^{14}$ of the T-QWR state were found. A confinement energy in T-shaped structures above $2 k_{B} \mathrm{~T}$ at room temperature has been realized by optimizing the structure parameters. ${ }^{15,16}$ Usage of $\operatorname{In}_{x} \mathrm{Ga}_{1-x}$ As layers at the $\mathrm{T}$ intersection was also shown to increase the confinement. ${ }^{17}$

In this paper, we investigate confinement-optimized asymmetric T-shaped quantum wires by transient four-wave mixing (FWM). The large confinement of the T-QWR exciton ground state, with respect to the exciton ground states of the intersecting QW's of more than $20 \mathrm{meV}$ in combination with a narrow inhomogeneous linewidth of $3.4 \mathrm{meV}$, makes it possible to determine the exciton and biexciton binding energies accurately. The exciton-exciton and exciton-phonon scattering are revealed in intensity and temperature dependences of the homogeneous linewidth determined by FWM.

\section{INVESTIGATED SAMPLE AND EXPERIMENTAL DETAILS}

The investigated sample contains asymmetric T-QWR's grown by MBE using the cleaved-edge overgrowth technique, ${ }^{6,9}$ as described in Refs. 15 and 18. It has a confinement-optimized asymmetry ${ }^{15}$ and consists of a 50period 24-nm [001] $\mathrm{Al}_{0.03} \mathrm{Ga}_{0.97} \mathrm{As} / 26-\mathrm{nm} \quad \mathrm{Al}_{0.3} \mathrm{Ga}_{0.7} \mathrm{As}$ multiple QW structure overgrown by a 6.6-nm [110] GaAs double QW, with 20-nm $\mathrm{Al}_{0.3} \mathrm{Ga}_{0.7}$ As barriers and a $15-\mathrm{nm}$ GaAs cap layer (see left inset in Fig. 1). The second overgrown QW is introduced as reference, since PL from the first overgrown QW is not observed due to the fast capture into the wire regions. The nonresonantly excited photolumines-

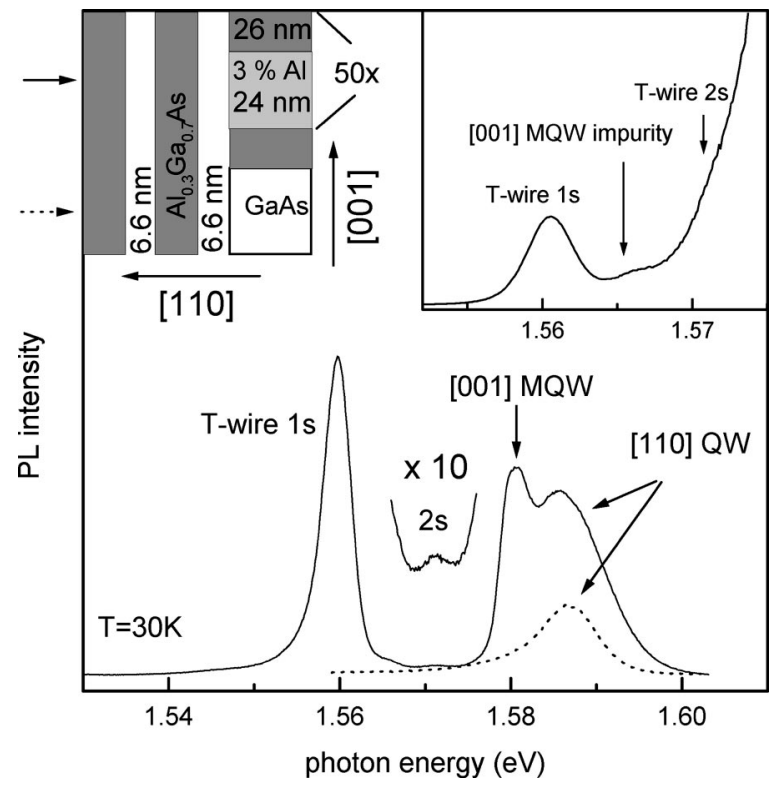

FIG. 1. PL spectra from the investigated T-QWR at $30 \mathrm{~K}$ lattice temperature and excitation at $1.96 \mathrm{eV}$ with $0.1 \mathrm{~W} / \mathrm{cm}^{2}$. The dotted line is the PL from the [110] reference QW, taken when exciting the GaAs substrate region. The left inset shows the sample structure, with the excitation positions indicated as arrows. The right inset shows the optical density calculated from the PL. The different lines are labeled. 
cence (PL) spectrum of the sample at $30 \mathrm{~K}$ (Fig. 1) shows in addition to the T-QWR $1 s$ exciton also the emission from the [001] MQW and the reference [110] QW. The reference QW emission is identified by the PL of the sample when excited in the region of the GaAs substrate (dotted line in Fig. 1). Even the $2 s$ state of the T-QWR exciton, which is identified by FWM, is visible in the PL as shoulder. Assuming a Boltzmann distribution of the carriers, we have calculated the optical density from the PL, shown in the right inset. The $1 \mathrm{~s}$ exciton shows a peak at $1.5606 \mathrm{eV}$ with a linewidth of 3.4 $\mathrm{meV}$. The $2 s$ resonance is covered by the tail of the [001] MQW signal and an impurity transition of the [001] MQW at $1.567 \mathrm{eV}$, which is identified in the PL of the nonovergrown structure. The data reveals an excellent ratio between more than $20 \mathrm{meV}$ confinement energy of the T-QWR exciton resonance and its inhomogeneous width.

We used transient FWM in reflection geometry with 100 300 -fs-long excitation pulses of $76 \mathrm{MHz}$ repetition rate generated by a self-mode-locked Ti:sapphire laser and spectrally shaped in a pulse shaper. The two excitation pulses of directions $\mathbf{k}_{1}$ and $\mathbf{k}_{2}$ and mutual delay time $\tau$ were focused on the sample by cylindrical optics to account for the small width of the T-wire array of only $2.5 \mu \mathrm{m}$. The resulting spot size was about $10 \times 70 \mu \mathrm{m}^{2}$. The FWM signal in the reflected $2 \mathbf{k}_{2}-\mathbf{k}_{1}$ direction was selected spatially by pinholes and detected time-integrated and spectrally resolved by a combination of a spectrometer and an optical multichannel analyzer. The excitation polarizations were adjusted by Babinet-Soleil compensators. In the following, linear polarization along the wires will be denoted by a vertical arrow.

\section{RESULTS AND DISCUSSION}

In this section we discuss separately the FWM results on the exciton localization, the exciton binding energy, the biexciton binding energy, the exciton-exciton scattering, and the exciton-phonon scattering.

\section{A. Exciton localization}

In semiconductor nanostructures, the disorder on the atomic scale, often classified as interface roughness and alloy fluctuations, creates a spatially varying potential for the confined excitons. This gives rise to exciton localization, ${ }^{19}$ which increases the exciton radiative lifetime, ${ }^{20}$ and leads to phonon-assisted relaxation into deeper localized exciton states (phonon-assisted hopping). ${ }^{21}$ Accordingly, the homogeneous exciton linewidth varies within the distribution of localized states, leading to increasing polarization decay times with decreasing energy. ${ }^{22-25}$ The phonon-assisted hopping has been observed in a $\mathrm{T}$-wire structure similar to the one investigated here by time-resolved PL. ${ }^{26}$ Using FWM, we determine the zero-density extrapolated homogeneous linewidths $\Gamma_{0}$ for various detection positions within the inhomogeneous line. In Fig. 2 the PL at $7 \mathrm{~K}$, the optical density, and the FWM spectrum at 1 ps delay time are displayed. The PL is Stokes shifted by $0.8 \mathrm{meV}$ relative to the optical density, $23 \%$ of the inhomogeneous broadening, which is significantly less than the $60 \%$ observed in QW structures. ${ }^{27}$ The reduced Stokes shift is a consequence of the restriction of the hopping to the one-dimensional movement along the $\mathrm{T}$ wire, which reduces the possible final states compared to the

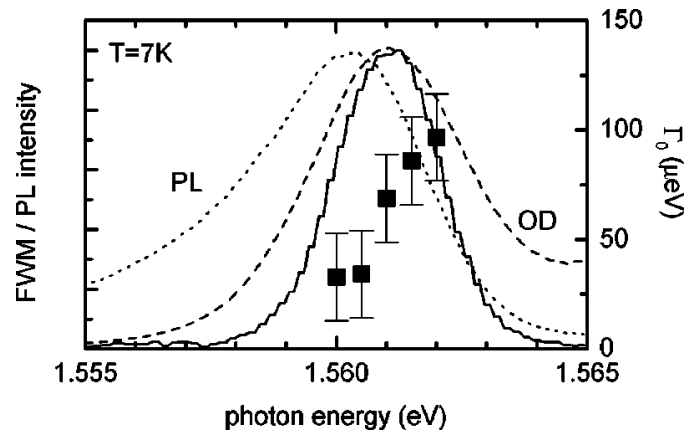

FIG. 2. Variation of the zero-density extrapolated homogeneous linewidth $\Gamma_{0}$ within the inhomogeneously broadened exciton transition at $7 \mathrm{~K}$. The FWM spectrum at $1 \mathrm{ps}$ delay time for collinear $(\uparrow \uparrow)$ excitation polarization, the PL (dotted line) and optical density (dashed line) spectra are given for comparison. The optical density (see Fig. 1) has been corrected for the temperature-induced bandgap shift at $30 \mathrm{~K}$ of about $0.5 \mathrm{meV}$.

QW case. ${ }^{28}$ The FWM linewidth of the $1 s$ exciton is 2.4 $\mathrm{meV}$, a factor of $\sqrt{2}$ smaller than its inhomogeneous broadening, as expected for a photon echo. ${ }^{29} \Gamma_{0}$ is decreasing with decreasing energy down to below $50 \mu \mathrm{eV}$. The radiative recombination time of about 350 ps (Ref. 26) gives a lower limit of $2 \mu \mathrm{eV}$ for the zero-temperature extrapolated $\Gamma_{0}$. From the analysis of the acoustic phonon scattering (see Sec. III E) we find that the stimulated acoustic phonon scattering at $7 \mathrm{~K}$ is expected to be $42 \pm 5 \mu \mathrm{eV}$. The increase of $\Gamma_{0}$ for higher energies is thus attributed to increasing spontaneous phonon-assisted relaxation to lower exciton states.

\section{B. Exciton binding energy}

The exciton binding energy in quantum wire structures is larger than in the bulk due to the two-dimensional confinement, as has been shown theoretically ${ }^{30}$ and experimentally. ${ }^{31,32}$ The complicated but well-controlled geometry of T-shaped quantum wires has stimulated wide interest in their exciton binding energy. The bound T-wire states are formed due to a decreased quantization energy in the intersecting region, which implies that the exciton binding energy in the $\mathrm{T}$ wire is not a priori larger than in the constituting QW's. Magneto-photoluminescence revealed a decreased exciton diameter in the [110] direction, ${ }^{13}$ and exciton binding energies of 25 (18) $\mathrm{meV}$ were deduced for symmetric $\quad 5$-nm GaAs/AlAs $\left(\mathrm{Al}_{0.3} \mathrm{Ga}_{0.7} \mathrm{As}\right) \quad$ T-shaped structure. ${ }^{33}$ Subsequent theoretical work found smaller binding energies. ${ }^{34-38}$ It was noticed that for structures with a large $\mathrm{Al}$ content in the barrier, the dielectric mismatch between well and barrier ${ }^{39,40}$ and the conduction-band nonparabolicity ${ }^{39}$ are important. Magnetophotoluminescence on confinement-optimized structures ${ }^{41}$ revealed moderate exciton binding energies of $11-13 \mathrm{meV}$, comparable to theoretical results. ${ }^{15,37}$

In FWM experiments, the exciton binding energy $E_{X}$ can be deduced from the $1 s-2 s$ energy splitting $E_{1 s-2 s}$, showing up as delay-time beats. ${ }^{42}$ This method was recently applied to etched $\operatorname{In}_{x} \mathrm{Ga}_{1-x}$ As quantum wires. ${ }^{43}$ We use here both the spectral and the delay-time information of the FWM data for simultaneous excitation of $1 s$ and $2 s$ resonances (Fig. 3). In 

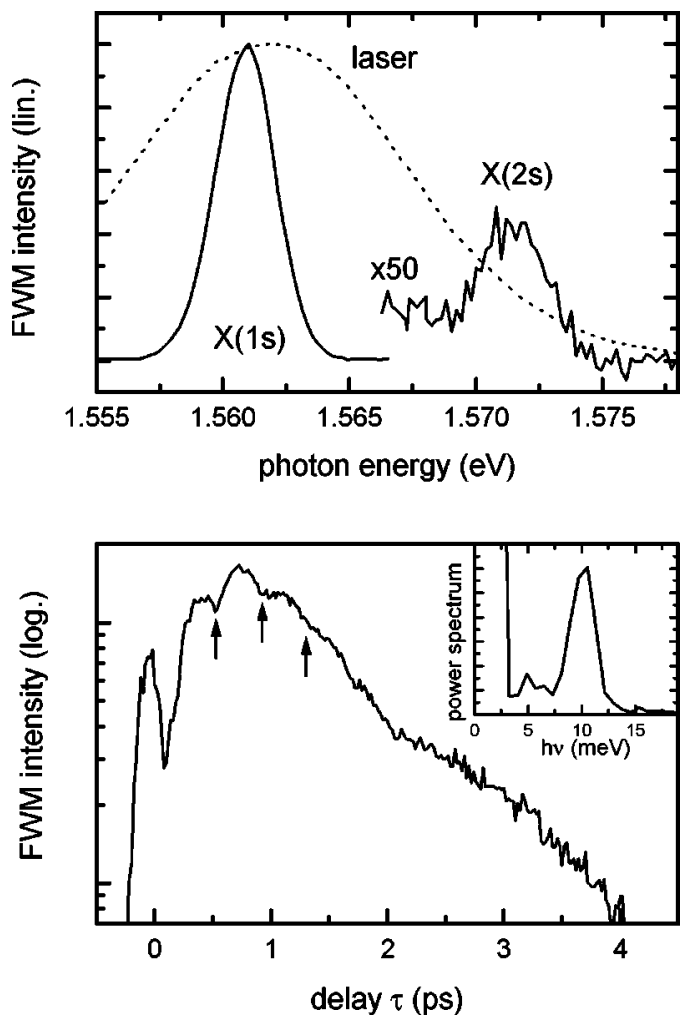

FIG. 3. FWM at $7 \mathrm{~K}$, an excitation density of about $7 \mu \mathrm{m}^{-1}$, and collinear $(\uparrow \uparrow)$ excitation polarization. Top: Spectra at a delay of $0.7 \mathrm{ps}$. The two excitonic peaks are indicated. The dotted line shows the laser spectrum. Bottom: FWM intensity versus delay time detected at a photon energy of $1.561 \mathrm{eV}$. The $1 s-2 s$ quantum beats are indicated by arrows. The inset shows the power spectrum of the Fourier-transformed intensity.

the FWM spectrum, a dominant response at the $1 s$ exciton transition and a spectral signature of the $2 s$ exciton 10.6 $\pm 0.4 \mathrm{meV}$ above are visible. The delay-time trace of the FWM intensity shows beats with a period of $400 \pm 20 \mathrm{fs}$, corresponding to an energy splitting of $10.4 \pm 0.5 \mathrm{meV}$. These beats identify the $2 s$ resonance, ${ }^{42}$ since they show a quantum-mechanical coupling to the $1 s$ state, and calculations show that the first excited state in the T-QWR is the $2 s$ state of the exciton. The longer beat period present is due to biexcitons as discussed in Sec. III C. $E_{1 s-2 s}$ is thus determined to $10.5 \pm 0.4 \mathrm{meV}$. To deduce $E_{X}$ from this splitting, we have to consider the exciton series in the $\mathrm{T}$ wire. For ideal three-, two-, or one-dimensional electron-hole relative motion, the ratio $E_{1 s-2 s} / E_{X}$ is $3 / 4,8 / 9$, and 1 , respectively. To describe a realistic quantum confined structure, the concept of a fractional dimensionality $\alpha$ for the relative motion has been introduced, ${ }^{44}$ for which the exciton series is given by

$$
E_{n}=-R_{y}^{*}\left(n+\frac{\alpha-3}{2}\right)^{-2}
$$

with the bulk exciton binding energy $R_{y}^{*}$. This determines $\alpha$ and $E_{X}$ to

$$
E_{1 s-2 s}=\frac{16 \alpha R_{y}^{*}}{\left(\alpha^{2}-1\right)^{2}}, \quad E_{X}=\frac{E_{1 s-2 s}(\alpha+1)^{2}}{4 \alpha}
$$

We apply this model to the investigated T-wire sample, for which we use $R_{y}^{*}=4.2 \mathrm{meV}$ from bulk GaAs. The effective dimensionality of the electron-hole relative motion in the $\mathrm{T}$-wire exciton is calculated to $\alpha=2.175 \pm 0.025$. The exciton binding energy is then deduced to $E_{X}=11.9$ $\pm 0.4 \mathrm{meV}$, in agreement with calculations for similar systems in the single subband approximation. ${ }^{15}$ More sophisticated calculations ${ }^{36,38,37}$ could not be directly compared due to the specific wire geometry, but similar values are obtained for comparable geometries. In the constituting QW's, the exciton binding energies are about $11 \mathrm{meV}$ for the $6.6 \mathrm{~nm}$ [110] and $7 \mathrm{meV}$ for the $24 \mathrm{~nm}$ [001] QW. The T-wire exciton binding energy in this asymmetric structure is thus slightly enhanced even compared to the narrow QW.

\section{Biexciton binding energy}

The formation of biexcitons with enhanced binding energies in low-dimensional semiconductors was first observed in quantum wells. ${ }^{45}$ Also in quantum wires, theory predicts a large enhancement of the biexciton binding. ${ }^{46,47}$ This was experimentally verified in etched $\mathrm{ZnSe}$ structures in the weak-confinement regime. ${ }^{48}$ Results on etched $\operatorname{In}_{x} \mathrm{Ga}_{1-x}$ As quantum wires ${ }^{49}$ showed that a repulsive Hartree potential between excitons due to differently confined electron and holes can counteract the confinement-induced enhancement. Additionally, the inhomogeneous broadening of the exciton transition can enhance the biexciton binding energy. ${ }^{50,51}$

FWM is suitable to observe and to identify the formation of biexcitons ${ }^{52}$ by polarization selection rules. ${ }^{25,53,54}$ FWM spectra and delay-time traces obtained from the T-QWR sample for different excitation polarizations are displayed in Fig. 4. The excitation pulse was spectrally shaped to excite only the $1 s$ exciton resonance. A biexcitonic FWM contribution is expected to be suppressed (but not forbidden due to the broken circular symmetry) for cocircular excitation, allowed but weaker than the excitonic FWM for collinear excitation, and significant due to the suppressed excitonic FWM for cross-linear excitation. ${ }^{53,55}$ The measured FWM spectra show such a behavior. The cocircular spectrum consists of a single peak at the exciton energy. For collinear polarization, a shoulder develops on the low-energy side, and for cross-linear polarization a low-energy peak dominates, while the exciton is suppressed. We accordingly attribute the low-energy peak to the exciton-biexciton polarization. The energy shift between the exciton peak and the biexciton peak of $2.0 \pm 0.2 \mathrm{meV}$ is the biexciton binding energy. The FWM delay-time traces support this interpretation. For cocircular polarization, we observe a single-exponential decay, while for collinear polarization a prominent beat with a period of $2.1 \pm 0.1$ ps appears, with a minimum at $\tau=0$, as expected for the exciton-biexciton polarization interference in inhomogeneously broadened systems. ${ }^{53,56}$ For cross-linear polarization, the beats show a $\pi$ phase shift as expected ${ }^{53}$ and are largely suppressed. This indicates that the exciton-unbound biexciton transition ${ }^{53,55}$ is of little importance. The faster signal decay for this polarization is attributed to an inhomogeneous broadening of the biexciton binding energy. ${ }^{56,55}$ The biexciton binding energy found from the beat period is 2.0 $\pm 0.1 \mathrm{meV}$. 

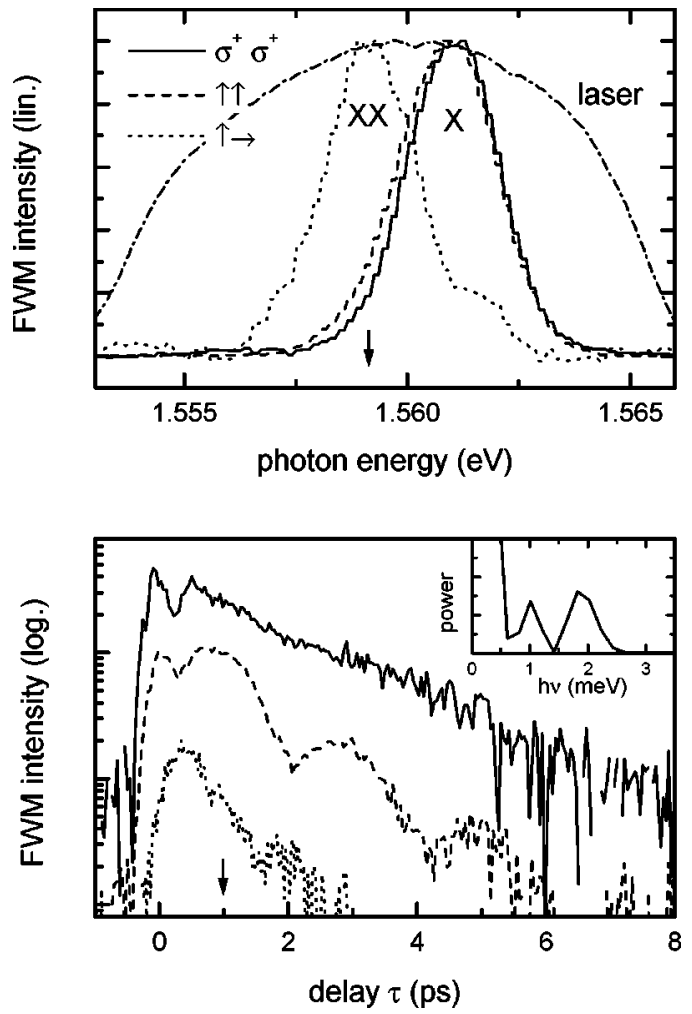

FIG. 4. FWM at $7 \mathrm{~K}$ and an exciton density of about $7 \mu \mathrm{m}^{-1}$ for the excitation polarizations cocircular $\left(\sigma^{+} \sigma^{+}\right)$(solid lines), collinear $(\uparrow \uparrow)$ (dashed lines), and cross linear $(\uparrow \rightarrow)$ (dotted lines). Top: Normalized spectra at a delay time $\tau=1$ ps (arrow in lower plot). The dash-dotted line is the laser spectrum. Bottom: Traces at a photon energy of $1.5595 \mathrm{eV}$ (arrow in upper plot). The data are vertically offset for clarity. The inset shows the power spectrum of the Fourier-transformed $(\uparrow \uparrow)$ intensity corrected by the overall dephasing.

The binding energy is significantly less than in $6 \mathrm{~nm}$ GaAs QW's of similar exciton binding energy and inhomogeneous width, for which about $3 \mathrm{meV}$ are found. ${ }^{54,51}$ Theory ${ }^{47}$ predicts $7 \mathrm{meV}$ binding energy for a rectangular GaAs quantum wire with dimensions $7 \times 25 \mathrm{~nm}^{2}$ and 11
meV exciton binding energy, comparable to the investigated structure. In etched $\operatorname{In}_{x} \mathrm{Ga}_{1-x}$ As quantum wires of $29 \mathrm{~nm}$ width and $12 \mathrm{meV}$ exciton binding energy, ${ }^{43}$ a biexciton binding energy of $2.1 \mathrm{meV}$ was found. ${ }^{49}$ The predicted strong enhancement of the biexciton binding energy is obviously not realized in the T-QWR and in the etched $\mathrm{In}_{x} \mathrm{Ga}_{1-x}$ As structure. Even more, the biexciton binding is less than in a QW of comparable exciton confinement. This indicates that the biexciton binding is reduced by the $e-h$ charge separation in the exciton due to their different confinements in the $\mathrm{T}$ wire, ${ }^{15,38}$ which introduces a repulsive exciton-exciton Hartree potential. Such a potential is not present for the confinement geometries considered in the theoretical descriptions. In the etched $\operatorname{In}_{x} \mathrm{Ga}_{1-x}$ As structures ${ }^{49}$ the same effect is present due to the penetration of the electrons into the GaAs barrier. ${ }^{57}$

\section{Exciton-exciton scattering}

With increasing exciton density, exciton-exciton scattering processes increase the homogeneous broadening of the exciton transition. An exciton-exciton scattering proportional to the exciton density was found in theory ${ }^{58}$ and in experiments on bulk GaAs (Ref. 59) and GaAs QW's. ${ }^{60}$. The corresponding homogeneous linewidth $\Gamma=2 \hbar / T_{2}$, with $T_{2}$ being the polarization decay time, can be expressed as

$$
\Gamma=\Gamma_{0}(T)+\Gamma_{X X}(T) N_{X}
$$

with the temperature-dependent zero-density dephasing $\Gamma_{0}(T)$, the exciton-exciton scattering parameter $\Gamma_{X X}(T)$, and the exciton density $N_{X}$. To compare with theory, ${ }^{58,61}$ $\Gamma_{X X}(T)$ can be normalized to the dimensionless scattering parameter $\gamma_{X X}(T)=\Gamma_{X X}(T) /\left(E_{X} a_{B}^{n}\right)$ using the exciton binding energy $E_{X}$, the Bohr radius $a_{B}$, and dimensionality $n$. Experimental values from literature on systems of different dimensionality are reported in Table I.

We determine the exciton-exciton scattering contribution to $\Gamma$ of the $1 s$ T-QWR exciton transition by intensitydependent FWM at $7 \mathrm{~K}$. The excitation polarization was collinear $(\uparrow \uparrow)$ along the wire. The exciton density was esti-

TABLE I. Exciton-exciton scattering parameters for structures of different materials and dimensionalities from literature. $\Gamma_{\text {inh }}:$ inhomogeneous broadening.

\begin{tabular}{|c|c|c|c|c|c|}
\hline Structure & $\Gamma_{\text {inh }}(\mathrm{meV})$ & $\mathrm{T}(\mathrm{K})$ & Excitation method & $\gamma_{X X}$ & $\Gamma_{X X}\left(\mu \mathrm{eV} \mu \mathrm{m}^{n}\right)$ \\
\hline 190 nm GaAs (3D) (Ref. 59) & $<1$ & 2 & nonresonant prepulse & 9 & 0.11 \\
\hline $80 \mathrm{~nm} \mathrm{ZnSe}$ (3D) (Ref. 80) & 4 & 5 & resonant prepulse & $19 \pm 6$ & $0.03 \pm 0.01$ \\
\hline 12 nm GaAs (2D) (Ref. 60) & 0.6 & 2 & nonresonant prepulse & $1.5 \pm 0.3$ & $1.2 \pm 0.02$ \\
\hline $8.5 \mathrm{~nm} \mathrm{CdTe}(2 \mathrm{D})($ Ref. 81$)$ & 5 & 5 & resonant FWM pulses & 0.16 & 0.065 \\
\hline $5 \mathrm{~nm} \mathrm{Zn}_{y} \mathrm{Cd}_{1-y} \mathrm{Se}$ (2D) (Ref. 24) & 6 & 10 & resonant FWM pulses & 0.17 & 0.063 \\
\hline $8 \mathrm{~nm} \mathrm{ZnSe}(2 \mathrm{D})($ Ref. 80$)$ & 5 & 5 & nonresonant prepulse & $5 \pm 2$ & $2 \pm 0.8$ \\
\hline $3 \mathrm{~nm} \operatorname{In}_{x} \mathrm{Ga}_{1-x} \mathrm{As}$ (2D) (Ref. 70) & 3 & 5 & resonant prepulse & 2 & 1.8 \\
\hline $1 \mathrm{~nm} \operatorname{In}_{x} \mathrm{Ga}_{1-x} \mathrm{As}$ (2D) (Ref. 65) & 0.7 & 5 & resonant FWM pulses & 0.3 & 0.25 \\
\hline $10 \times 60 \mathrm{~nm}^{2}$ GaAs etched (1D) (Ref. 25) & $?$ & 8 & resonant FWM pulses & $5-11$ & $500-1200$ \\
\hline $16 \times 23 \mathrm{~nm}^{2} \mathrm{ZnSe}$ etched (1D) (Ref. 73) & 4 & 5 & resonant FWM pulses & $15 \pm 5$ & $1400 \pm 400$ \\
\hline $3 \times 29 \mathrm{~nm}^{2} \quad \operatorname{In}_{x} \mathrm{Ga}_{1-x}$ As etched (1D) (Ref. 70) & 4 & 5 & resonant prepulse & 19 & 230 \\
\hline $7 \times 24 \mathrm{~nm}^{2}$ GaAs $\mathrm{T}$ (1D) (this work) & 3 & 7 & resonant FWM pulses & 0.2 & 26 \\
\hline
\end{tabular}



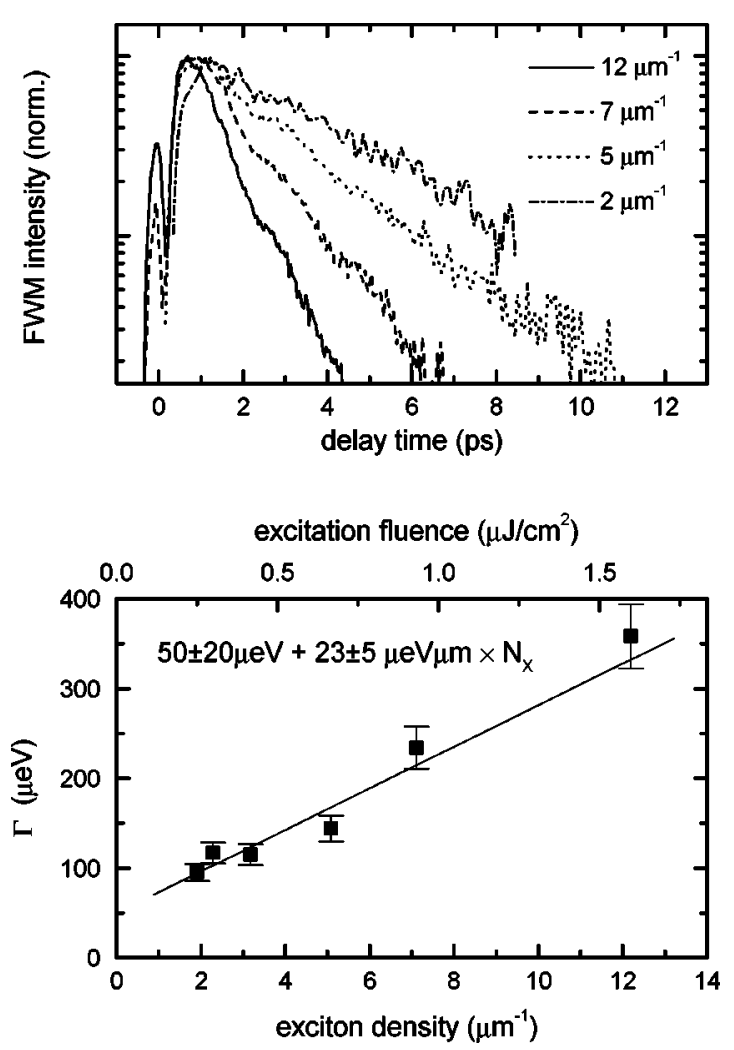

FIG. 5. FWM at the T-wire exciton at $7 \mathrm{~K}$ for collinear ( $\uparrow \uparrow)$ polarization and different excitation densities, as indicated. Excitation spectrum as in Fig. 4. Top: Normalized FWM intensity at $1.5611 \mathrm{eV}$ versus delay time. Bottom: Deduced homogeneous broadening $\Gamma$ versus excitation intensity, and linear fit to the data.

mated from the excitation intensity and the exciton absorption into the wire of 24-nm width. For the integrated absorption probability, the value of $0.165 \mathrm{meV}$ for quasitwo-dimensional excitons in 6-nm GaAs single QW's ${ }^{62}$ which have a comparable exciton binding energy, was used. The polarization anisotropy of the T-wire absorption ${ }^{15,14}$ leads to an enhancement of the oscillator strength of about $20 \%$ for the linear polarization along the wire. The intensity of the $k_{2}$ pulse has been weighted by a factor of $1 / 2$ since it only influences the polarization decay between the arrival of $k_{2}$ and the emission of the photon echo. The homogeneous linewidth is then deduced from the decay time $T_{2} / 4$ of the photon-echo intensity with pulse delay, as shown in Fig. 5. A linear fit to the data by Eq. (3) gives $\Gamma_{0}=50 \pm 20 \mu \mathrm{eV}$ and $\Gamma_{X X}=23 \pm 5 \mu \mathrm{eV} \mu \mathrm{m} . \Gamma_{0}$ is attributed to acoustic-phonon scattering, as discussed in Secs. III A and III E. A dimensionless scattering parameter $\gamma_{X X}=0.2 \pm 0.02$ is calculated from $\Gamma_{X X}$ using the exciton binding energy of $12 \mathrm{meV}$ determined in Sec. III B, and the corresponding exciton radius of $11 \mathrm{~nm}$ obtained from the fractional-dimensional model and $n=1$. Both $\Gamma_{X X}$ and $\gamma_{X X}$ are significantly smaller than previously reported on etched quantum wire structures (see Table I). Strongly varying scattering parameters are also reported for QW structures and have been tentatively attributed to exciton localization. However, there is no clear correlation between reported inhomogeneous linewidth and scattering parameter. The different excitation conditions, which are providing differently thermalized exciton distributions, and the uncertain determination of the exciton density, could be other sources

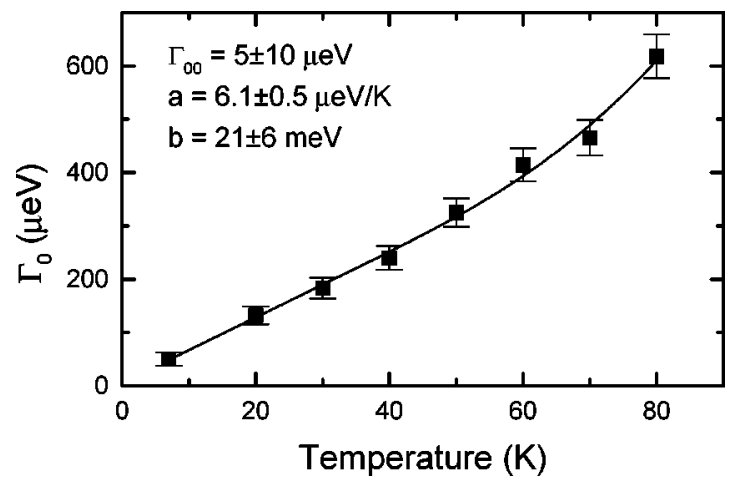

FIG. 6. Zero-density extrapolated homogeneous linewidths of the T-wire exciton at the center of the inhomogeneous distribution $(1.5611 \mathrm{eV}$ at $5 \mathrm{~K})$ as a function of lattice temperature. The line is a fit by Eq. (4), with the parameters as indicated.

of systematic errors. Also, the creation of unpaired free carriers in the quantum structures, which can be observed by the formation of charged excitons, ${ }^{63,64}$ can modify ${ }^{65}$ the observed density dependence.

\section{E. Exciton-phonon scattering}

The exciton-phonon scattering was investigated widely in bulk and quantum-well structures. The temperature dependence of the low-density exciton homogeneous broadening due to acoustic and optical-phonon scattering can be expressed by ${ }^{66}$

$$
\Gamma_{0}(T)=\Gamma_{00}+a T+\frac{b}{\exp \left(\hbar \omega_{\mathrm{LO}} / k_{B} T\right)-1}
$$

with the temperature-independent contribution $\Gamma_{00}$, the acoustic-phonon coefficient $a$, and the optical-phonon coefficient $b$. The temperature-dependences of the two contributions result from the occupation number of the respective phonons given by the Bose-Einstein distribution. The linear dependence for the acoustic phonons stems from the assumption that the energies of the interacting acoustic phonons, which are restricted by momentum conservation, are less than the thermal energy $k_{B} T$. In confined systems, the upper limit for the phonon energy is determined by the smallest confinement length and is typically below $1 \mathrm{meV}$ in GaAs QW's. The inhomogeneous broadening $\Gamma_{\text {inh }}$ of the excitons leads to a localization of the excitons, which can change the temperature dependence for $k_{B} T<\Gamma_{\text {inh }}$ into an activated behavior. ${ }^{67,68}$ In spite of this, one can often find a linear increase of $\Gamma_{0}$ with temperature even in samples with large $\Gamma_{\text {inh. }}{ }^{69,70}$ We determine $\Gamma_{0}(T)$ by intensity-dependent FWM, as described in Sec. III D. The experimental data for $\Gamma_{0}(T)$ are shown in Fig. 6 as a function of the lattice temperature, and are fitted by Eq. (4), using the GaAs LOphonon energy of $\hbar \omega_{\mathrm{LO}}=36 \mathrm{meV}$. The resulting opticalphonon coefficient $b=21 \pm 6 \mathrm{meV}$ is comparable to values found in GaAs QW's (Ref. 71) and quantum wires. ${ }^{72} \mathrm{~A}$ large effect of the one-dimensional confinement on $b$ is not expected since the confinement energies are less than the LOphonon energy. The determined acoustic-phonon scattering coefficient is $a=6.1 \pm 0.5 \mu \mathrm{eV} / \mathrm{K}$. For comparison results on GaAs and $\mathrm{In}_{x} \mathrm{Ga}_{1-x} \mathrm{As}$, QW's from literature are shown 


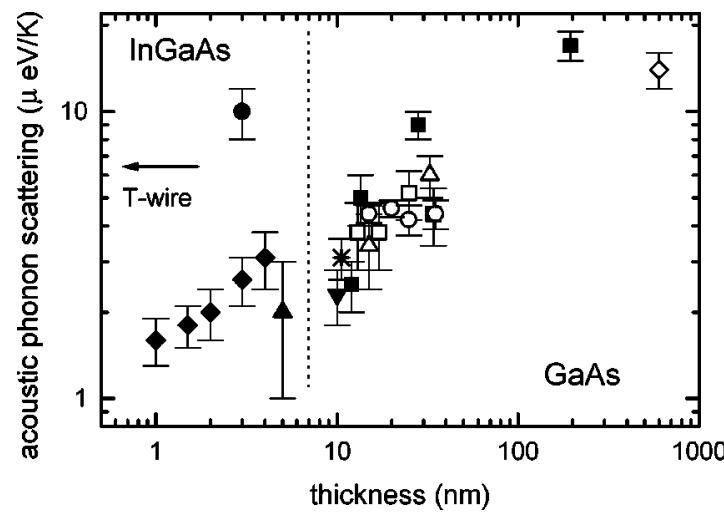

FIG. 7. Acoustic-phonon scattering coefficients $a$ for GaAs and $\mathrm{In}_{x} \mathrm{Ga}_{1-x}$ As layers versus layer thickness from literature. FWM results: full symbols [squares (Ref. 74), circle (Ref. 43), diamonds (Ref. 65), up triangle (Ref. 75), down triangle (Ref. 76), and star (Ref. 25)]. Line-shape analysis results: open symbols [squares (Ref. 71), circles (Ref. 77), up triangle (Ref. 78), and diamond (Ref. 79)]. The arrow indicates the value found in the present work.

in Fig. 7. The present result is about twice the value obtained for $10 \mathrm{~nm} \mathrm{GaAs}$ QW's or $4 \mathrm{~nm} \mathrm{In}_{x} \mathrm{Ga}_{1-x}$ As QW's, which have a comparable exciton binding energy. This indicates that the additional confinement direction increases the phonon interaction due to the relaxed momentum conservation in the phonon scattering. Such a behavior is predicted for quasi-two-dimensional systems, where $a$ should scale inversely to the size of the exciton in the confinement direction, which was reported for $\mathrm{In}_{x} \mathrm{Ga}_{1-x}$ As QW's. ${ }^{65}$ For GaAs QW's, however, no systematic dependence is found. For etched quantum wires, $6.2 \pm 0.5 \mu \mathrm{eV} / \mathrm{K}$ in $60 \times 10 \mathrm{~nm}^{2}$ $\mathrm{GaAs}$ wires $^{25}$ and $12 \pm 0.5 \mu \mathrm{eV} / \mathrm{K}$ in etched 23 $\times 3 \mathrm{~nm}^{2} \operatorname{In}_{x} \mathrm{Ga}_{1-x}$ As wires ${ }^{43}$ were found. In the latter structure $10 \pm 0.5 \mu \mathrm{eV} / \mathrm{K}$ were found for the reference $\mathrm{QW}$, much higher than reported in Ref. 65 for a comparable structure.
The zero-temperature and zero-density extrapolated homogeneous linewidth $\Gamma_{00}$ is $5 \pm 10 \mu \mathrm{eV}$, much lower than reported in etched wires, ${ }^{25,43,73}$ and shows the absence of extrinsic effects like surface trapping. A lower limit to $\Gamma_{00}$ of about $2 \mu \mathrm{eV}$ is given by the radiative decay, as discussed in Sec. III A.

\section{CONCLUSION}

We have investigated the properties of excitons in a GaAs T-shaped quantum wire with more than $20 \mathrm{meV}$ confinement energy and $3.4 \mathrm{meV}$ inhomogeneous broadening. The exciton binding energy of $12 \mathrm{meV}$ is in agreement with theoretical predictions. The biexciton binding energy of $2 \mathrm{meV}$ is less than theoretically expected and indicates a repulsive exciton-exciton interaction due to different electron and hole confinement. The inhomogeneous broadening leads to exciton localization and phonon-assisted relaxation between the localized states, as shown by time-resolved photoluminescence, photoluminescence Stokes shift, and decreasing homogeneous linewidth with deeper localization within the exciton distribution. A dimensionless exciton-exciton scattering parameter of $\gamma_{X X}=0.2$ is found, much smaller than previously reported on etched quantum wires. The exciton acoustic-phonon interaction of $6 \mu \mathrm{eV} / \mathrm{K}$ is about a factor of 2 larger than in comparable QW structures, which can be due to the relaxed momentum conservation along the second confinement direction.

\section{ACKNOWLEDGMENTS}

We want to thank C. B. Sørensen for expert assistance in the MBE growth and J. R. Jensen and P. Borri for helpful discussions. This work was supported by the Center for Nanostructures (CNAST).
*Present address: Lehrstuhl für Experimentelle Physik EIIb, Universität Dortmund, Otto-Hahn Str.4, D-44221 Dortmund, Germany.

${ }^{\dagger}$ Present address: Farœse Telecom, P.O. Box 27, FO-110 Torshavn, Farœ Islands.

${ }^{\ddagger}$ Present address: Research Center COM, The Technical University of Denmark, Bldg. 349, DK-2800 Lyngby, Denmark.

${ }^{1}$ P. Ils, M. Michel, A. Forchel, I. Gyuro, M. Klenk, and E. Zielinski, Appl. Phys. Lett. 64, 496 (1994).

${ }^{2}$ M. S. Miller, H. Weman, C. E. Pryor, M. Krishnamurthy, P. M. Petroff, H. Kroemer, and J. L. Merz, Phys. Rev. Lett. 68, 3464 (1992).

${ }^{3}$ E. Kapon, K. Kash, E. M. Jr. Clausen, D. M. Hwang, and E. Colas, Appl. Phys. Lett. 60, 477 (1992).

${ }^{4}$ Y.-C. Chang, L. L. Chang, and L. Esaki, Appl. Phys. Lett. 47, 1324 (1985).

${ }^{5}$ L. Pfeiffer, K. W. West, H. L. Stormer, J. P. Eisenstein, K. W. Baldwin, D. Gershoni, and J. Spector, Appl. Phys. Lett. 56, 1697 (1990).

${ }^{6}$ D. Gershoni, I. Brener, G. A. Baraff, S. N. G. Chu, L. N. Pfeiffer, and K. West, Phys. Rev. Lett. 65, 1631 (1990).

${ }^{7}$ H. L. Störmer, L. N. Pfeiffer, K. W. Baldwin, K. W. West, and J.
Spector, Appl. Phys. Lett. 58, 726 (1991).

${ }^{8}$ A. Goñi, L. N. Pfeiffer, K. W. West, A. Pinczuk, H. U. Baranger, and H. L. Stormer, Appl. Phys. Lett. 61, 1956 (1992).

${ }^{9}$ W. Wegscheider, L. N. Pfeiffer, A. Pinczuk, K. W. West, M. M. Dignam, R. Hull, and R. E. Leibenguth, J. Cryst. Growth 150, 285 (1995).

${ }^{10}$ R. D. Grober, T. D. Harris, J. K. Trautman, E. Betzig, W. Wegscheider, L. Pfeiffer, and K. West, Appl. Phys. Lett. 64, 1421 (1994).

${ }^{11}$ W. Wegscheider, L. N. Pfeiffer, M. M. Dignam, A. Pinczuk, K. W. West, S. L. McCall, and R. Hull, Phys. Rev. Lett. 71, 4071 (1993).

${ }^{12}$ W. Wegscheider, L. N. Pfeiffer, M. Dignam, A. Pinczuk, K. W. West, and R. Hull, Semicond. Sci. Technol. 9, 1933 (1994).

${ }^{13}$ T. Someya, H. Akiyama, and H. Sakaki, Phys. Rev. Lett. 74, 3664 (1995).

${ }^{14}$ H. Akiyama, T. Someya, and H. Sakaki, Phys. Rev. B 53, R10 520 (1996).

${ }^{15}$ W. Langbein, H. Gislason, and J. M. Hvam, Phys. Rev. B 54, 14 595 (1996)

${ }^{16}$ H. Gislason, W. Langbein, and J. M. Hvam, Appl. Phys. Lett. 69, 3248 (1996). 
${ }^{17}$ H. Akiyama, T. Someya, M. Yoshita, T. Sasaki, and H. Sakaki, Phys. Rev. B 57, 3765 (1998).

${ }^{18}$ H. Gislason, C. Sørensen, and J. M. Hvam, Appl. Phys. Lett. 69, 800 (1996).

${ }^{19}$ R. Zimmermann, F. Große, and E. Runge, Pure Appl. Chem. 69, 1179 (1997).

${ }^{20}$ A. V. Kavokin, Phys. Rev. B 50, 8000 (1994).

${ }^{21}$ H. Kalt, J. Collet, S. D. Baranowskii, R. Saleh, P. Thomas, L. S. Dang, and J. Cibert, Phys. Rev. B 45, 4253 (1992).

${ }^{22}$ H. Schwab, V. G. Lyssenko, and J. M. Hvam, Phys. Rev. B 44, 3413 (1991).

${ }^{23}$ M. Koch, D. Weber, J. Feldmann, E. O. Göbel, T. Meier, A. Schulze, P. Thomas, and S. Schmitt-Rink, Phys. Rev. B 47, 1532 (1993).

${ }^{24}$ A. J. Fischer, D. S. Kim, J. Hays, W. Shan, J. J. Song, D. B. Eason, J. Ren, J. F. Schetzina, H. Luo, and J. K. Furdyna, Phys. Rev. B 50, 17643 (1994).

${ }^{25}$ E. J. Mayer, G. O. Smith, V. Heukenroth, J. Kuhl, K. Bott, A. Schulze, T. Meier, D. Bennhardt, S. W. Koch, and P. Thomas, Phys. Rev. B 50, 14730 (1994).

${ }^{26}$ H. Gislason, W. Langbein, and J. M. Hvam, Superlattices Microstruct. 22, 217 (1997).

${ }^{27}$ F. Yang, M. Wilkinson, E. J. Austin, and K. P. O’Donnel, Phys. Rev. Lett. 70, 323 (1993).

${ }^{28}$ L. E. Golub, E. L. Ivchenko, and A. A. Kiselev, J. Opt. Soc. Am. B 13, 1199 (1996)

${ }^{29}$ J. Erland, K. H. Pantke, V. Mizeikis, V. G. Lyssenko, and J. M. Hvam, Phys. Rev. B 50, 15047 (1994).

${ }^{30}$ M. H. Degani and O. Hipólito, Phys. Rev. B 35, 9345 (1987).

${ }^{31}$ Y. Nagamune, Y. Arakawa, S. Tsukamoto, M. Nishioka, S. Sasaki, and N. Miura, Phys. Rev. Lett. 69, 2963 (1992).

${ }^{32}$ E. Rinaldi, R. Cingolani, M. Lepore, M. Ferrera, I. M. Catalano, F. Rossi, L. Rota, E. Molinari, P. Lugli, U. Marti, D. Martin, F. Morier-Gemond, P. Ruterana, and F. Reinhart, Phys. Rev. Lett. 73, 2899 (1994).

${ }^{33}$ T. Someya, H. Akiyama, and H. Sakaki, Phys. Rev. Lett. 76, 2965 (1996).

${ }^{34}$ G. W. Bryant, P. S. Julienne, and Y. B. Band, Superlattices Microstruct. 20, 602 (1996).

${ }^{35}$ D. Brinkmann and G. Fishman, Phys. Rev. B 56, 15211 (1997).

${ }^{36}$ S. N. Walck, T. L. Reinecke, and P. A. Knipp, Phys. Rev. B 56, 9235 (1997).

${ }^{37}$ F. Rossi, G. Goldoni, and E. Molinari, Phys. Rev. Lett. 78, 3527 (1997).

${ }^{38}$ S. Glutsch, F. Bechstedt, W. Wegscheider, and G. Schedelbeck, Phys. Rev. B 56, 4108 (1997).

${ }^{39}$ S. Glutsch, F. Bechstedt, W. Wegscheider, and G. Schedelbeck, Phys. Status Solidi A 164, 405 (1997).

${ }^{40}$ G. Goldoni, F. Rossi, and E. Molinari, Phys. Rev. Lett. 80, 4995 (1998).

${ }^{41}$ W. Langbein, H. Gislason, J. M. Hvam, and J. Zeman, IQEC Technical Digest (Optical Society of America, Washington D.C., 1998), Vol. 7, p. 87.

${ }^{42}$ J. Feldmann, T. Meier, G. V. Plessen, M. Koch, E. O. Göbel, P. Thomas, G. Bacher, C. Hartmann, H. Schweizer, W. Schäfer, and H. Nickel, Phys. Rev. Lett. 70, 3027 (1993).

${ }^{43}$ W. Braun, M. Bayer, A. Forchel, H. Zull, J. P. Reithmaier, A. I. Filin, S. N. Walck, and T. L. Reinecke, Phys. Rev. B 55, 9290 (1997).

${ }^{44}$ P. Lefebvre, P. Christol, and H. Mathieu, Phys. Rev. B 48, 17308 (1993).
${ }^{45}$ R. Miller, D. Kleinman, A. Gossard, and O. Munteanu, Phys. Rev. B 25, 6545 (1982).

${ }^{46}$ L. Bányai, I. Galbraith, C. Ell, and H. Haug, Phys. Rev. B 36, 6099 (1987).

${ }^{47}$ F. Madarasz, F. Szmulowicz, F. Hopkins, and D. Dorsey, Phys. Rev. B 49, 13528 (1994).

${ }^{48}$ H. P. Wagner, W. Langbein, J. M. Hvam, G. Bacher, T. Kümmell, and A. Forchel (unpublished).

${ }^{49}$ T. Baars, W. Braun, M. Bayer, and A. Forchel, Phys. Rev. B 58, R1750 (1998).

${ }^{50}$ W. Langbein and J. M. Hvam, Phys. Status Solidi B 206, 111 (1998).

${ }^{51}$ W. Langbein and J. M. Hvam, Phys. Rev. B 59, 15405 (1999).

${ }^{52}$ K. H. Pantke, D. Oberhauser, V. G. Lyssenko, J. M. Hvam, and G. Weimann, Phys. Rev. B 47, 2413 (1993).

${ }^{53}$ T. F. Albrecht, K. Bott, T. Meier, A. Schulze, M. Koch, S. T. Cundiff, J. Feldmann, W. Stolz, P. Thomas, S. W. Koch, and E. O. Göbel, Phys. Rev. B 54, 4436 (1996).

${ }^{54}$ S. Adachi, T. Miyashita, S. Takeyama, Y. Takagi, A. Tackeuchi, and M. Nakayama, Phys. Rev. B 55, 1654 (1997).

${ }^{55} \mathrm{~W}$. Langbein, P. Borri, and J. Hvam, in Radiative Processes and Dephasing in Semiconductors, edited by D. Citrin (Optical Society of America, Washington D.C., 1998), Vol. 18, p. 11.

${ }^{56}$ W. Langbein, J. M. Hvam, M. Umlauff, H. Kalt, B. Jobst, and D. Hommel, Phys. Rev. B 55, R7383 (1997).

${ }^{57}$ J. P. Reithmaier, R. Höger, and H. Riechert, Phys. Rev. B 43, 4933 (1991).

${ }^{58}$ F. Boldt, K. Henneberger, and V. May, Phys. Status Solidi B 130, 675 (1985).

${ }^{59}$ L. Schultheis, J. Kuhl, A. Honold, and C. W. Tu, Phys. Rev. Lett. 57, 1635 (1986).

${ }^{60}$ A. Honold, L. Schultheis, J. Kuhl, and C. W. Tu, Phys. Rev. B 40, 6442 (1989).

${ }^{61}$ G. Manzke, K. Henneberger, and V. May, Phys. Status Solidi B 139, 233 (1987).

${ }^{62}$ W. T. Masselink, P. J. Pearah, J. Klem, C. K. Peng, H. Morkoç, G. D. Sanders, and Y.-C. Chang, Phys. Rev. B 32, 8027 (1985).

${ }^{63}$ K. Kheng, R. T. Cox, Y. Merle d'Aubigné, F. Bassani, K. Saminadayar, and S. Tatarenko, Phys. Rev. Lett. 71, 1752 (1993).

${ }^{64}$ S. Glasberg, G. Finkelstein, H. Shtrikman, and I. Bar-Joseph, Phys. Rev. B 59, R10 425 (1999).

${ }^{65}$ P. Borri, W. Langbein, J. M. Hvam, and F. Martelli, Phys. Rev. B 59, 2215 (1999).

${ }^{66}$ S. Rudin, T. L. Reinecke, and B. Segall, Phys. Rev. B 42, 11218 (1990).

${ }^{67}$ D. Gammon, E. S. Snow, B. V. Shanabrook, D. S. Katzer, and D. Park, Science 277, 85 (1996).

${ }^{68}$ M. D. Webb, S. T. Cundiff, and D. G. Steel, Phys. Rev. B 43, 12 658 (1991).

${ }^{69}$ I. J. Blewett, D. J. Bain, A. Tookey, G. Brown, I. Galbraith, A. K. Kar, B. Vögele, K. A. Prior, B. C. Cavenett, and B. S. Wherrett, Phys. Rev. B 59, 9756 (1999).

${ }^{70}$ W. Braun, M. Bayer, A. Forchel, O. M. Schmitt, L. Bańyai, H. Haug, and A. I. Filin, Phys. Rev. B 57, 12364 (1998).

${ }^{71}$ D. Gammon, S. Rudin, T. L. Reinecke, D. S. Katzer, and C. S. Fyono, Phys. Rev. B 51, 16785 (1995).

${ }^{72}$ A. V. Gopal, R. Kumar, A. S. Vengurlekar, T. Mélin, F. Laruelle, and B. Etienne, Appl. Phys. Lett. 74, 2474 (1999).

${ }^{73}$ H. P. Wagner, W. Langbein, J. M. Hvam, G. Bacher, T. Kümmell, and A. Forchel, Phys. Rev. B 57, 1797 (1998). 
${ }^{74}$ J. Kuhl, A. Honold, L. Schultheis, and C. W. Tu, Festkörperprobleme 29, 157 (1989).

${ }^{75}$ G. V. M. Williams, C. C. Phillips, and K. Woodbridge, Semicond. Sci. Technol. 9, 1096 (1994).

${ }^{76}$ D.-S. Kim, J. Shah, J. E. Cunningham, T. C. Damen, W. Schäfer, M. Hartmann, and S. Schmitt-Rink, Phys. Rev. Lett. 68, 1006 (1992).

${ }^{77}$ W. Langbein (private communication).

${ }^{78}$ V. Srinivas, J. Hryniewicz, Y. J. Chen, and C. E. C. Wood, Phys.
Rev. B 46, 10193 (1992).

${ }^{79}$ A. Tredicucci, Y. Chen, F. Bassani, J. Massies, C. Deparis, and G. Neu, Phys. Rev. B 47, 10348 (1993).

${ }^{80}$ H. P. Wagner, A. Schätz, R. Maier, W. Langbein, and J. M. Hvam, Phys. Rev. B 56, 12581 (1997).

${ }^{81}$ R. Hellmann, M. Koch, J. Feldmann, S. T. Cundiff, E. O. Göbel, D. R. Yakovlev, A. Waag, and G. Landwehr, Phys. Rev. B 48, 2847 (1993). 